Published in final edited form as:

J Am Geriatr Soc. 2015 September ; 63(9): 1880-1885. doi:10.1111/jgs.13593.

\title{
Associations of Mild Cognitive Impairment with Hospitalization and Readmission
}

\author{
Kathryn E. Callahan, MD, MS ${ }^{1}$, James F. Lovato, MS $^{2}$, Michael E. Miller, $\mathrm{PhD}^{2}$, Doug \\ Easterling, $\mathrm{PhD}^{3}$, Beth Snitz, $\mathrm{PhD}^{4}$, and Jeff D. Williamson, MD, MHS. ${ }^{1}$ \\ ${ }^{1}$ Wake Forest School of Medicine, Department of Internal Medicine, Section on Gerontology and \\ Geriatric Medicine and the Sticht Center on Aging, Winston-Salem, North Carolina \\ ${ }^{2}$ Wake Forest Health Sciences, Division of Public Health Sciences, Department of Biostatistical \\ Sciences, Winston-Salem, North Carolina
}

Corresponding Author: Kathryn E. Callahan, MD, MS, Department of Internal Medicine, Section on Gerontology and Geriatric Medicine, Wake Forest School of Medicine, Medical Center Boulevard, Winston-Salem, North Carolina 27157,

kecallah@wakehealth.edu, T: 336-713-8583 F: 336-716-0427.

Alternate Corresponding Author: jwilliam@wakehealth.edu

Requests for reprints should be sent to: Kathryn E. Callahan, MD, MS, Department of Internal Medicine, Wake Forest School of Medicine, Medical Center Boulevard, Winston-Salem, North Carolina, 27157, kecallah@wakehealth.edu

Conflict of Interest Checklist:

\begin{tabular}{|l|c|c|c|c|c|c|c|c|c|c|c|c|c|}
\hline $\begin{array}{l}\text { Elements of } \\
\text { Financial/Personal } \\
\text { Conflicts }\end{array}$ & \multicolumn{2}{|l|}{ Callahan } & \multicolumn{2}{l}{ Lovato } & \multicolumn{2}{l|}{ Miller } & \multicolumn{2}{l|}{ Easterling } & \multicolumn{2}{l|}{ Snitz } & \multicolumn{2}{l|}{ Williamson } \\
\hline & Yes & No & Yes & No & Yes & No & Yes & No & Yes & No & Yes & No \\
\hline Employment or Affiliation & & $\mathrm{X}$ & & $\mathrm{X}$ & & $\mathrm{X}$ & & $\mathrm{X}$ & & $\mathrm{X}$ & & $\mathrm{X}$ \\
\hline Grants/Funds & & $\mathrm{X}$ & & $\mathrm{X}$ & & $\mathrm{X}$ & & $\mathrm{X}$ & & $\mathrm{X}$ & & $\mathrm{X}$ \\
\hline Honoraria & & $\mathrm{X}$ & & $\mathrm{X}$ & & $\mathrm{X}$ & & $\mathrm{X}$ & & $\mathrm{X}$ & & $\mathrm{X}$ \\
\hline Speaker Forum & & $\mathrm{X}$ & & $\mathrm{X}$ & & $\mathrm{X}$ & & $\mathrm{X}$ & & $\mathrm{X}$ & & $\mathrm{X}$ \\
\hline Consultant & & $\mathrm{X}$ & & $\mathrm{X}$ & & $\mathrm{X}$ & & $\mathrm{X}$ & & $\mathrm{X}$ & & $\mathrm{X}$ \\
\hline Stocks & & $\mathrm{X}$ & & $\mathrm{X}$ & & $\mathrm{X}$ & & $\mathrm{X}$ & & $\mathrm{X}$ & & $\mathrm{X}$ \\
\hline Royalties & & $\mathrm{X}$ & & $\mathrm{X}$ & & $\mathrm{X}$ & & $\mathrm{X}$ & & $\mathrm{X}$ & & $\mathrm{X}$ \\
\hline Expert Testimony & & $\mathrm{X}$ & & $\mathrm{X}$ & & $\mathrm{X}$ & & $\mathrm{X}$ & & $\mathrm{X}$ & & $\mathrm{X}$ \\
\hline Board Member & & $\mathrm{X}$ & & $\mathrm{X}$ & & $\mathrm{X}$ & & $\mathrm{X}$ & & $\mathrm{X}$ & & $\mathrm{X}$ \\
\hline Patents & & $\mathrm{X}$ & & $\mathrm{X}$ & & $\mathrm{X}$ & & $\mathrm{X}$ & & $\mathrm{X}$ & & $\mathrm{X}$ \\
\hline Personal Relationship & & $\mathrm{X}$ & & $\mathrm{X}$ & & $\mathrm{X}$ & & $\mathrm{X}$ & & $\mathrm{X}$ & & $\mathrm{X}$ \\
\hline
\end{tabular}

For "yes", provide a brief explanation: not applicable

Author Contributions:

Analysis: Dr. Callahan, Mr. Lovato, and Dr. Miller had full access to all of the data in the study and take responsibility for the integrity of the data and the accuracy of the data analysis.

Study concept and design: Callahan, Lovato, Miller, Easterling, Snitz, Williamson

Acquisition of data: Williamson, Snitz, and the GEMS study group

Analysis and interpretation of data: Callahan, Lovato, Miller, Easterling, Williamson

Drafting of the manuscript: Callahan, Williamson, Miller, Easterling

Critical revision of the manuscript for important intellectual content: Callahan, Lovato, Miller, Easterling, Snitz, Williamson

Administrative, technical, or material support: Callahan, Lovato, Miller, Easterling, Williamson 
${ }^{3}$ Wake Forest Health Sciences, Division of Public Health Sciences, Department of Social Sciences and Health Policy, Winston-Salem, North Carolina

${ }^{4}$ University of Pittsburgh, Department of Neurology, Pittsburgh, Pennsylvania

\section{Abstract}

OBJECTIVES-To determine whether older adults with mild cognitive impairment (MCI), a condition not previously explored as a risk factor, experience increased hospitalizations and 30day readmission compared to those with normal cognition. Frequent hospitalizations and unplanned readmissions are recognized as markers of poor quality care for older adults.

DESIGN-Post-hoc analysis of prospectively gathered data on incident hospitalization and readmission from the Ginkgo Evaluation of Memory Study (GEMS), a randomized double-blind placebo-controlled trial designed to assess the impact of Ginkgo biloba on incidence of dementia.

SETTING-GEMS was conducted in 5 academic medical centers in the United States.

PARTICIPANTS-2742 community-dwelling adults age 75 or older with normal cognition $(\mathrm{n}=2314)$ or MCI $(\mathrm{n}=428)$ at baseline cognitive testing.

MEASUREMENTS-Index hospitalization and 30-day hospital readmission, adjusted for age, sex, race, education, clinic site, trial assignment status, comorbidities, number of prescription medications, and living with an identified proxy.

RESULTS-MCI was associated with a $17 \%$ increase in the hazard of index hospitalization as compared with normal cognition (adjusted Hazard Ratio (HR)=1.17 (1.02 - 1.34)). In participants who lived with their proxy, MCI was associated with a $39 \%$ increased hazard of index hospitalization (adjusted HR=1.392 (1.169 - 1.657)). Baseline MCI was not associated with increased odds of 30-day hospital readmission (adjusted Odds Ratio=0.90 $(0.60-1.36)$ ).

CONCLUSION-MCI may represent a target condition for healthcare providers to coordinate support services in an effort to reduce hospitalization and subsequent disability.

\section{Keywords}

rehospitalization; mild cognitive impairment (MCI); acute hospital utilization

\section{INTRODUCTION}

Adults 65 years and older represent $13 \%$ of the United States population and, given their higher disease burden, consume $43 \%$ of healthcare spending on hospitalizations. ${ }^{1}$ The Centers for Medicare and Medicaid Services (CMS) increasingly emphasizes quality care at reasonable cost, with increased scrutiny on high cost care settings like inpatient hospitals. In this context, unplanned hospital readmissions are markers of poor quality care. ${ }^{2,3}$ One in five hospitalized Medicare beneficiaries will be readmitted within 30 days, ${ }^{4}$ increasing their risk for hospitalization-associated disability, ${ }^{5}$ while incurring high costs to the healthcare system ( $\$ 17.5$ billion in 2010). ${ }^{4}$ 
Healthcare systems researchers have identified diagnosis, ${ }^{6}$ demographics (age, race, ethnicity, and gender), ${ }^{7}$ and social determinants of health (socioeconomic status, caregiver support, marital status, and health literacy $)^{8}$ as risk factors for hospitalization and readmission. However, risk prediction models rarely include cognitive impairment, a factor with strong justification for being predictive of excess hospitalization. ${ }^{9,10}$

Cognitive impairment may refer to dementia or to its often unrecognized precursor: mild cognitive impairment (MCI). MCI may be defined through low scores on neuropsychological testing - without functional impairment. ${ }^{11} \mathrm{MCI}$, which often goes unrecognized, may impair executive function or working memory; older adults with MCI may struggle with self-management of chronic disease, ${ }^{12}$ which would suggest that MCI increases the risk of hospitalization and readmission. Hospitalization and acute care does occur more frequently in older adults with dementia, ${ }^{12-14}$ but though MCI is associated with increased costs, ${ }^{15}$ studies have not yet explored MCI as a predictor for hospitalization or readmission.

The Ginkgo Evaluation of Memory Study (GEMS) provides an excellent database of well characterized participants, including a subset with MCI, to explore the association of cognition with hospitalization. GEMS enrolled 3072 community-dwelling adults over 75 years old to test the efficacy of Ginkgo biloba in preventing or delaying Alzheimer's Disease, through a double-blind randomized placebo-controlled clinical trial. ${ }^{16}$ GEMS researchers classified participants into MCI and normal cognition groups, utilizing neuropsychological testing and functional surveys. ${ }^{17}$ Using these MCI definitions from GEMS, we aimed to determine a) whether older adults classified with $\mathrm{MCI}$ at the baseline cognitive assessment experience increased rates of index hospitalization (participants' first hospitalization after baseline assessment) compared to those with normal cognition; b) among older adults with an index hospitalization, whether those with MCI are at greater risk of 30-day readmission; and c) whether living with a proxy modifies the effect of MCI on hospitalization and readmission. We hypothesized that those with MCI would experience increased rates of index hospitalization and 30-day readmission compared with those with normal cognition. Social support is associated with reduced risk of hospital utilization and readmission in older adults with presumed normal cognition. ${ }^{7,8,18}$ We anticipated that living with an identified proxy would modify the effect of MCI on these outcomes.

\section{METHODS}

\section{Study Population and Study Design}

The GEMS protocols are reported previously. ${ }^{16,17,19}$ Participants gave informed consent, and participants and proxies reported participants' physical and cognitive function and follow up data, including hospitalizations. ${ }^{19}$ Of note, GEMS excluded participants with known dementia, depression, and those taking medications for dementia; upon initial screening, participants were excluded with Modified Mini Mental State Exam (3MSE) ${ }^{20}$ scores $<80 / 100$, or with Center for Epidemiological Studies Depression (CES-D) Scale $>14 .{ }^{21}$ Prior to randomization, participants underwent comprehensive physical, neurologic, and neuropsychiatric evaluation. Baseline medical history and medications were collected. Regularly scheduled follow up visits occurred wherein participants and proxies reported 
major health events, including hospitalizations; researchers reviewed hospital paperwork. Individuals who reached the dementia endpoint were excluded from further follow up, including hospitalizations. Total mean follow-up was six years. For these post-hoc analyses, we excluded participants with missing baseline demographics, medications, past medical history, or proxy information; after application of these criteria, 2742 participants were included. The GEMS study demonstrated no impact of Ginkgo biloba on memory; ${ }^{19}$ therefore, we combined placebo and treatment groups for these analyses.

\section{Predictor: $\mathrm{MCl}$}

Our predictor was MCI status at baseline. In GEMS, participants completed ten neuropsychological tests across five cognitive domains typically tested for dementia: verbal and visual memory; language; attention and psychomotor speed; executive functions; and visuospatial construction. If scores in two or more domains were more than 1.5 standard deviations below Cardiovascular Health Study (CHS) established age- and education-level means, potential participants were again excluded. Clinical Dementia Rating (CDR) scores ${ }^{22}$ were collected from prospective participants' proxies. Participants completed the cognitive portion of the Alzheimer's Disease Assessment Scale (ADAS-Cog) ${ }^{23}$

Once the researchers defined a cohort of non-demented participants, guidelines from the International Working Group for MCI were utilized to identify baseline MCI cases using the tests above; this process has been described elsewhere. ${ }^{11,17}$ Criteria included: a) CDR global score of 0.5 , indicating no more than minimal impairment in complex instrumental functions; b) a less than 10th percentile score on at least two of the ten cognitive tests performed during screening. These definitions were selected to minimize false positives. Thus, participants were identified at baseline as having MCI $(n=480)$ or normal cognition $(\mathrm{n}=2592)$. At each follow up appointment, participants completed the 3MSE, CDR, and ADAS-Cog. Scores indicating a possible incident case of dementia triggered a complete neuropsychological battery and subsequent adjudication of dementia diagnoses. Incident MCI was not identified during follow up.

\section{Living with Others}

As social support has demonstrated an independent effect on hospitalization and readmission, we included living with a proxy as a covariate. However, older adults with MCI and declining cognition could be more likely to have a live-in proxy, and a proxy could influence an older adult with MCI to seek hospital care. Therefore, we also considered living with a proxy as a potential effect modifier in the relationship between MCI and index hospitalization, and between MCI and 30-day hospital readmission.

\section{Outcomes: Hospitalization and Hospital Readmission}

Our outcomes were rates of index hospitalization and 30-day hospital readmission, defined as a hospital admission occurring within 30 days of discharge from the index hospitalization. Participants and proxies reported medical history and hospitalizations at follow up. Discharge summaries were collected and reviewed for dates of hospital admission, discharge, and primary and secondary diagnoses. For the 30-day readmission analysis, we 
included participants who had experienced and survived an index hospitalization through 30 days following hospital discharge $(\mathrm{n}=1627)$.

\section{Covariates}

Covariates relating to $\mathrm{MCI}$ and hospitalization/readmission included demographic factors (age, sex, race, education), baseline comorbidities (hypertension, diabetes, cancer, congestive heart failure, myocardial infarction, angina, kidney disease, liver disease, stroke, emphysema), number of prescription medications, and clinic site (to address geographic variation in hospitalization). ${ }^{24}$ As GEMS did not demonstrate impact of Ginkgo biloba, we tested for heterogeneity of the relationship between MCI and hospitalization/readmission (unreported analyses) among randomized groups, combined the data, and included randomization group status as a covariate. ${ }^{16,19}$

\section{Statistical Analyses}

Baseline demographics, clinic site, Ginkgo biloba status, comorbidities, prescription medications, and social support were described using simple proportions; differences between baseline MCI and normal cognition groups for each descriptor were compared using t-tests for continuous variables and chi-square for categorical variables.

Chi-square analyses were used to compare the proportions of index hospitalization, total number of hospitalizations, 30-day readmissions, development of dementia, and death within each group. We conducted a priori calculations for power, determining that this dataset would yield $82 \%$ power to detect a $25 \%$ increased risk of an index hospitalization in the MCI group versus those with normal cognition.

Proportional hazards regression analyses were used to model the relationship between baseline MCI status and time from baseline assessment to index hospitalization. Participants who completed the study without an index hospitalization were censored with a time value equal to the number of days between their baseline and final GEMS follow-up visit. Participants who died prior to experiencing hospitalization were censored. Our initial round of analyses censored those who died prior to index hospitalization at the time of death. In a second set of analyses, we combined mortality with index hospitalization (hospitalizationfree survival). Since conclusions were unchanged, we present the censored models. The proportionality assumption was tested by including a time-varying covariate of MCI group*time interaction in the fully adjusted model. This term was not significant ( $p=0.73$ ). First, an unadjusted model was fit; the second model included age, sex, race, education, clinic site, and trial assignment status (Ginkgo biloba versus placebo). Sequential models added baseline comorbidities; number of prescription medications; and whether participants lived with their listed proxy. Finally, we tested for an interaction between MCI at baseline and living with a proxy within the full model. Results are presented as Hazard Ratios (HR) with 95\% confidence intervals (CI) relating the hazard for hospitalization for those with $\mathrm{MCI}$ at baseline to the same hazard in those with normal cognition.

For 30-day readmissions, we conducted logistic regression analyses to model the relationship between MCI status at baseline and 30-day readmission. We adjusted for 
potential confounders, including demographics, clinic site, Ginkgo biloba status, comorbidities, number of prescription medications, living with a proxy, and interaction between MCI and living with a proxy. A priori calculation for power showed that the available readmissions data yielded $81 \%$ power to detect a 30 -day readmission rate of $16 \%$ in participants with $\mathrm{MCI}$ as compared with a rate of $10 \%$ for those with normal cognition. We explored whether the odds ratio relating readmission to baseline MCI status varied by trial assignment status (Ginkgo biloba versus placebo) using the Breslow Day statistic; this did not reach statistical significance, so we did not stratify analyses by trial assignment. Results are presented as odds ratios (OR) with 95\% confidence intervals relating the odds of readmission within 30 days for those with $\mathrm{MCI}$ at baseline compared with those without.

\section{RESULTS}

\section{Baseline Characteristics}

Mean age of all GEM participants at baseline was 78.6 years; $46 \%$ were female, and $96 \%$ were white. Over $60 \%$ had some college education, and $23 \%$ completed graduate work. Participants with MCI at baseline were more likely to be older, female, and of minority race (Table 1), more likely to have a history of myocardial infarction, angina, and stroke, and more likely to self-report their health as fair or poor. No differences in baseline comorbidity appeared between those who lived with a proxy versus those who did not (unreported analyses).

\section{Index Hospitalization}

There were 1688 participants who experienced at least one hospitalization during follow up (61\%), and 307 (18.19\%) experienced four or more hospitalizations (Table 1). After adjusting for all covariates, MCI conferred a $17 \%$ increase in the rate of index hospitalizations as compared with participants with normal cognition (unadjusted Hazard Ratio (HR)=1.245 (1.091 - 1.420); adjusted HR=1.170 (1.021 - 1.342); Table 2). Median time to index hospitalization was 43 months for those with MCI $(35,50)$ compared with 50 months $(48,53)$ for normal cognition.

We found a significant interaction between MCI and living with a proxy (chi square 8.274, $\mathrm{p}=0.004$ ). In participants who lived with their proxy, MCI was associated with a $39 \%$ increase in the rate of index hospitalization compared with those with normal cognition (adjusted HR 1.392, 1.169 - 1.657, Table 2). However, in participants who did not live with their proxy, there was no relationship between MCI and the rate of index hospitalization (adjusted HR 0.936, $0.758-1.155$ ). Table 3 displays hazard ratios for index hospitalization, compared across $\mathrm{MCI}$ and living with proxy statuses.

\section{0-Day Hospital Readmission}

We investigated the relationship between MCI and 30-day hospital readmission. After excluding the 61 participants who did not survive to 30 days following discharge, there were 1627 participants eligible for readmission. We found no significant difference in the proportion of 30-day readmissions by baseline MCI status; $13.5 \%$ of participants with MCI experienced a 30-day readmission as compared with $11.7 \%$ of those with normal cognition 
$(\mathrm{p}=0.423)$. MCI was not associated with an increased odds of 30-day readmission in any model investigated (fully adjusted OR $0.902,0.598-1.363$ ). The interaction between MCI status and living with a proxy for 30-day readmission was not statistically significant $(\mathrm{p}=0.103)$.

\section{DISCUSSION}

In community-dwelling older adults, MCI was associated with a $17 \%$ increase in index hospitalization. We hypothesized that MCI would increase hospitalization, as those with MCI struggle with complex tasks necessary for self-care. Since social support reduces hospitalizations and readmissions, we hypothesized that MCI would increase hospitalization in those without a live-in proxy. However, the inverse was shown: in those participants who lived with their proxy, MCI increased the rate of index hospitalization by $39 \%$, while not impacting participants who did not live with a proxy (adjusted HR 0.936, $0.758-1.155$ ). This finding holds implications for health care access for those with MCI: older adults with MCI living alone may delay hospitalization, lacking the insight to recognize "red flags" of illness; alternatively, those with live-in proxies may recognize warning signs of impending illness and access acute care more promptly. Regardless, the interaction between MCI and living with a proxy suggests that MCI status could reflect older adults who may over- or under-use hospital services, depending on their degree of support. Therefore, screening for MCI may identify patients and caregivers who could benefit from intensive care coordination.

To our knowledge, this analysis within a large clinical trial is one of the first studies with geographically diverse, well-defined cognitive phenotypes to address the impact of MCI on hospitalization; prior studies on hospital utilization ${ }^{13}$ or hospital readmissions ${ }^{12,14}$ have emphasized dementia, or single cognitive tests. In this study, MCI was determined on the basis of comprehensive neuropsychiatric testing, ${ }^{17}$ and conducted prior to hospitalization, which minimizes confounding with delirium. Outcomes of hospitalization and readmission were clearly defined, carefully ascertained by study staff, and relevant to current policy definitions. However, it is important to acknowledge the study's methodological and database limitations. As with most clinical trials, GEMS is not representative of the general population in racial, socioeconomic, and educational diversity, which limits generalizability, as participants may have had greater access to services. The original GEMS study excluded participants with depression, whose risk for increased hospital services is recognized. In addition, there were no measurements of Overall readmission (12\%) was lower than expected in the general population. ${ }^{1}$ The null findings for readmission may reflect limited power to detect a clinically meaningful result due to a relatively small sample size. Finally, once the adjudicated dementia endpoint for GEMS was achieved, participants were no longer followed for events. This decision within GEMS may have excluded a small number of outcomes.

This analysis rigorously examines a novel source of risk by utilizing a clearly defined, research-based, expert definition of MCI. Of the population over 75, over $20 \%$ is estimated to have MCI. ${ }^{25}$ As described by Fried et al. for early physical impairment, ${ }^{26} \mathrm{MCI}$ may represent the "bottom of the iceberg," an often unrecognized impairment that still confers 
increased risk for an older adult's hospitalization and subsequent disability. ${ }^{5,27}$ Therefore future research could explore whether screening for MCI - currently a rare practice - may identify a subgroup of older adults and their caregivers who would benefit from ambulatory care coordination, caregiver support, and community-based services to reduce recurrent hospitalizations.

\section{Acknowledgments}

We acknowledge the participating institutions and principal staff of the GEM Study, of which this study is a part: from the National Center for Complementary and Alternative Medicine: Richard L. Nahin, Barbara Sorkin; from the John Hopkins University: Linda Fried, Michelle Carlson, Pat Crowley, Claudia Kawas, Paulo Chaves, and Joyce Chabot; from the University of California-Davis: John Robbins, Katherine Gundling, Sharene Theroux, and Linly Kwong; from the University of Pittsburgh: Lewis Kuller, Roberta Moyer, and Cheryl Albig; from the Wake Forest University School of Medicine: Gregory L Burke, Steve Rapp, Dee Posey, and Margie Lamb; from the Data Coordinating Center at the University of Washington, Seattle: Dick Kronmal, Annette Fitzpatrick, Fumei Lin, Cam Solomon, and Alice Arnold; from the Cognitive Diagnostic Center at the University of Pittsburgh: Steven T. DeKosky, Judith Saxton, Oscar Lopez, Diane Ives, and Leslie Dunn; from the Clinical Coordinating Center at the Wake Forest University School of Medicine: Curt Furberg, Jeff Williamson, Nancy Woolard, Kathryn Bender, and Susan Margitić; from the Central Laboratory at the University of Vermont: Russell Tracy, Elaine Cornell, Nancy Jenny; from the MRI Reading Center at the University of Pittsburgh: Carolyn Meltzer; from Schwabe Pharmaceuticals, Germany: Robert Hörr, Thorsten Schmeller and Joachim Herrmann; from the Data Safety Monitoring Board: Richard Grimm (Chair), Jonathan Berman (Executive Secretary), Hannah Bradford, Carlo Calabrese, Rick Chappell, Kathryn Connor; Gail Geller, Boris Iglewicz, Richard S. Panush, and Richard Shader.

Many thanks to Ms. Laura Hayworth for help with formatting. Thank you to the Wake Forest University Health Sciences Masters Program in Clinical and Population Translational Science. Finally, our sincere thanks to Stephen Kritchevsky, $\mathrm{PhD}$, for review of the manuscript.

Funding Source: The original Ginkgo Evaluation of Memory Study was funded by Grant Number 5 U01 AT000162 from the National Center for Complementary and Alternative Medicine (NCCAM) and the Office of Dietary Supplements, and support from the National Institute on Aging, National Heart, Lung, and Blood Institute, the University of Pittsburgh Alzheimer's Disease Research Center (P50AG05133), the Roena Kulynych Center for Memory and Cognition Research, and National Institute of Neurological Disorders and Stroke.

Dr. Callahan has received support from the Wake Forest University Claude D. Pepper Older Americans Independence Center (P30-AG21332), the John A. Hartford Foundation, Department of Health and Human Services (DHHS), Health Resources and Services Administration (HRSA), Bureau of Health Professions (BHPr), under Geriatric Academic Career Award K01HP20490, The contents are solely the responsibility of the authors and should not be construed as the official position or policy of, nor should any endorsements be inferred by the DHHS, HRSA, BHPR, NCCAM, NIH or the U.S. Government.

Sponsor's role: The John A. Hartford Foundation, HRSA, the Pepper OAIC and the NIA/NCCAM did not take part in the design and conduct of the study; the collection, management, analysis, and interpretation of the data; preparation, review, or approval of this manuscript; or the decision to submit the manuscript for publication.

\section{References}

1. Agency for Healthcare Research and Quality. Healthcare cost and utilization project facts and figures. 2008

2. Williams MV. A requirement to reduce readmissions: take care of the patient, not just the disease. JAMA. 2013; 309:394-396. [PubMed: 23340642]

3. Kocher RP, Adashi EY. Hospital readmissions and the Affordable Care Act: paying for coordinated quality care. JAMA. 2011; 306:1794-1795. [PubMed: 22028355]

4. Jencks SF, Williams MV, Coleman EA. Rehospitalizations among patients in the Medicare fee-forservice program. N Engl J Med. 2009; 360:1418-1428. [PubMed: 19339721]

5. Covinsky KE, Palmer RM, Fortinsky RH, et al. Loss of independence in activities of daily living in older adults hospitalized with medical illnesses: increased vulnerability with age. J Am Geriatr Soc. 2003; 51:451-458. [PubMed: 12657063] 
6. Kansagara D, Englander H, Salanitro A, et al. Risk prediction models for hospital readmission: a systematic review. JAMA. 2011; 306:1688-1698. [PubMed: 22009101]

7. Shulan M, Gao K, Moore CD. Predicting 30-day all-cause hospital readmissions. Health Care Manag Sci. 2013; 16:167-175. [PubMed: 23355120]

8. Arbaje AI, Wolff JL, Yu Q, et al. Postdischarge environmental and socioeconomic factors and the likelihood of early hospital readmission among community-dwelling Medicare beneficiaries. Gerontologist. 2008; 48:495-504. [PubMed: 18728299]

9. Naylor MD, Hirschman KB, Bowles KH, et al. Care coordination for cognitively impaired older adults and their caregivers. Home Health Care Serv Q. 2007; 26:57-78. [PubMed: 18032200]

10. Piraino E, Heckman G, Glenny C, et al. Transitional care programs: who is left behind? A systematic review. Int J Integr Care. 2012; 12:e132. [PubMed: 23593046]

11. Winblad B, Palmer K, Kivipelto M, et al. Mild cognitive impairment--beyond controversies, towards a consensus: report of the International Working Group on Mild Cognitive Impairment. J Intern Med. 2004; 256:240-246. [PubMed: 15324367]

12. Buslovich S, Kennedy GJ. Potential effect of screening for subtle cognitive deficits on hospital readmission. J Am Geriatr Soc. 2012; 60:1980-1981. [PubMed: 23057454]

13. Callahan CM, Arling G, Tu W, et al. Transitions in care for older adults with and without dementia. J Am Geriatr Soc. 2012; 60:813-820. [PubMed: 22587849]

14. Dodson JA, Truong TT, Towle VR, et al. Cognitive impairment in older adults with heart failure: prevalence, documentation, and impact on outcomes. Am J Med. 2013; 126:120-126. [PubMed: 23331439]

15. Zhu CW, Sano M, Ferris SH, et al. Health-related resource use and costs in elderly adults with and without mild cognitive impairment. J Am Geriatr Soc. 2013; 61:396-402. [PubMed: 23414481]

16. DeKosky ST, Fitzpatrick A, Ives DG, et al. The Ginkgo Evaluation of Memory (GEM) study: design and baseline data of a randomized trial of Ginkgo biloba extract in prevention of dementia. Contemp Clin Trials. 2006; 27:238-253. [PubMed: 16627007]

17. Snitz BE, Saxton J, Lopez OL, et al. Identifying mild cognitive impairment at baseline in the Ginkgo Evaluation of Memory (GEM) study. Aging Ment Health. 2009; 13:171-182. [PubMed: 19347684]

18. Landi F, Onder G, Cesari M, et al. Comorbidity and social factors predicted hospitalization in frail elderly patients. J Clin Epidemiol. 2004; 57:832-836. [PubMed: 15551473]

19. Snitz BE, O'Meara ES, Carlson MC, et al. Ginkgo biloba for preventing cognitive decline in older adults: a randomized trial. JAMA. 2009; 302:2663-2670. [PubMed: 20040554]

20. Teng EL, Chui HC. The Modified Mini-Mental State (3MS) examination. J Clin Psychiatry. 1987; 48:314-318. [PubMed: 3611032]

21. Radloff LS. The CES-D scale: a self-report depression scale for research in the general population. Applied Psychological Measurement. 1977; 1:16.

22. Morris JC. The Clinical Dementia Rating (CDR): current version and scoring rules. Neurology. 1993; 43:2412-2414. [PubMed: 8232972]

23. Rosen WG, Mohs RC, Davis KL. A new rating scale for Alzheimer's disease. The American journal of psychiatry. 1984; 141:1356-1364. [PubMed: 6496779]

24. Fisher ES, Wennberg JE, Stukel TA, et al. Hospital readmission rates for cohorts of Medicare beneficiaries in Boston and New Haven. N Engl J Med. 1994; 331:989-995. [PubMed: 8084356]

25. Pusswald G, Moser D, Gleiss A, et al. Prevalence of mild cognitive impairment subtypes in patients attending a memory outpatient clinic--comparison of two modes of mild cognitive impairment classification. Results of the Vienna Conversion to Dementia Study. Alzheimer's \& dementia : the journal of the Alzheimer's Association. 2013; 9:366-376.

26. Fried LP, Herdman SJ, Kuhn KE, et al. Preclinical Disability: Theories about the Bottom of the Iceberg. Journal of Aging and Health. 1991:285-300.

27. Sager MA, Franke T, Inouye SK, et al. Functional outcomes of acute medical illness and hospitalization in older persons. Arch Intern Med. 1996; 156:645-652. [PubMed: 8629876] 


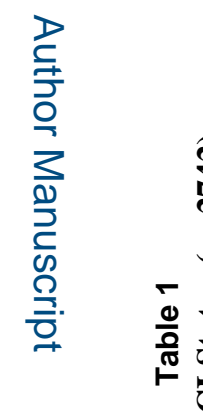

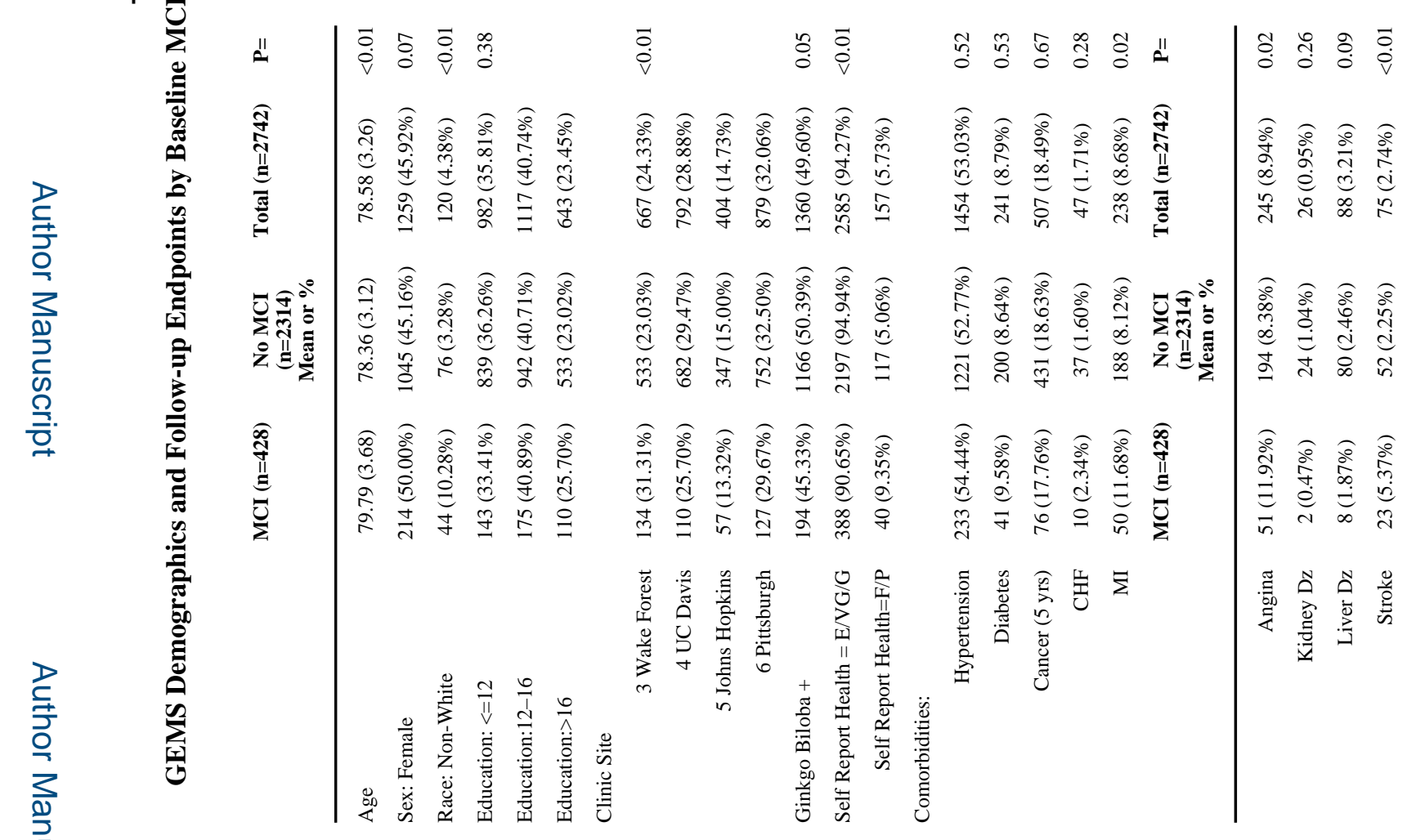




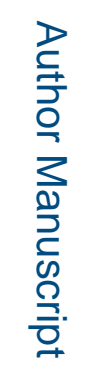

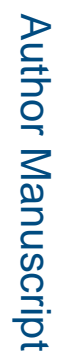

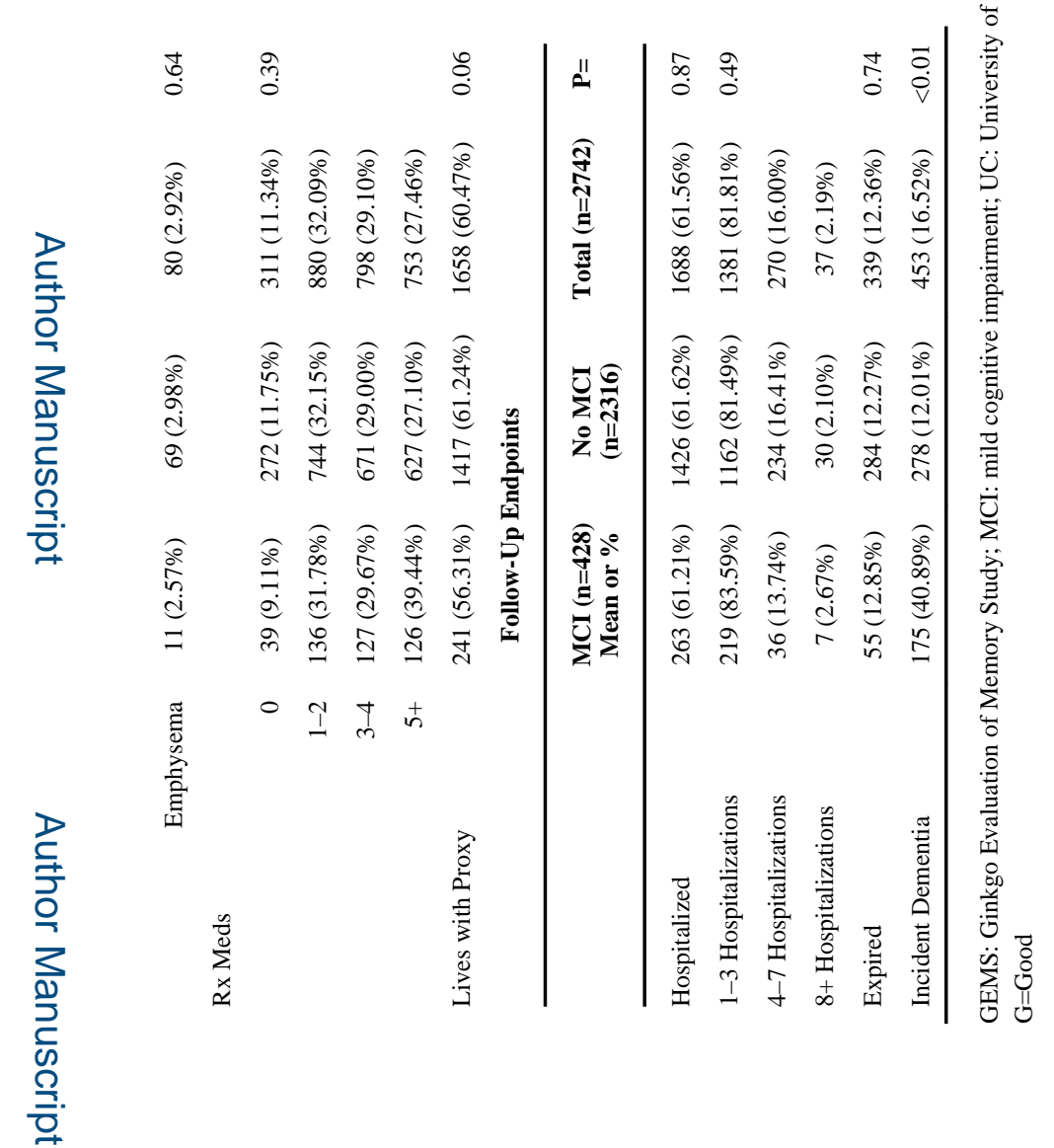

J Am Geriatr Soc. Author manuscript; available in PMC 2016 March 28. 
Table 2

\section{Hazard Ratios for Index Hospitalization Comparing Mild Cognitive Impairment (MCI)} Status to Normals at Baseline

The reference group is those with normal cognition.

\begin{tabular}{|c|c|c|c|}
\hline Model & HR & $95 \% \mathrm{CI}$ & $\mathbf{P}$ \\
\hline Unadjusted & 1.25 & $1.09-1.42$ & $<0.01$ \\
\hline + demographics, clinic site, Ginkgo biloba assignment & 1.22 & $1.07-1.40$ & $<0.01$ \\
\hline Above + comorbidities & 1.19 & $1.04-1.37$ & 0.01 \\
\hline Above + number of prescription medications & 1.18 & $1.03-1.35$ & 0.02 \\
\hline Above + plus living with proxy & 1.17 & $1.02-1.34$ & 0.02 \\
\hline Above, in participants living with proxy ${ }^{*}$ & 1.39 & $1.17-1.66$ & $\mathrm{n} / \mathrm{a}$ \\
\hline Above, in participants not living with proxy ${ }^{*}$ & 0.94 & $0.76-1.16$ & $\mathrm{n} / \mathrm{a}$ \\
\hline
\end{tabular}

Legend: Demographics include age, sex, race, level of education. Comorbidities: hypertension, diabetes, cancer, congestive heart failure, myocardial infarction, angina, kidney disease, liver disease, stroke, emphysema. "Living with proxy" refers to whether a participant lives with the listed proxy.

* The interaction of MCI at baseline and living with proxy was significant (chi-square 8.274, $\mathrm{p}=0.004$ ), therefore the final model is presented stratified by whether the participant lives with proxy. 
Table 3

Comparison of Hazard Ratios for Index Hospitalization Comparing Mild Cognitive Impairment (MCI) Status and Living with Proxy status to Reference

The reference group is those with normal cognition who live with their identified proxy.

\begin{tabular}{lll}
\hline & Does Not Live with Proxy & Lives with Proxy \\
MCI & 1.07 & $\mathbf{1 . 3 9}$ \\
Normal Cognition & $\mathbf{1 . 1 4}$ & 1.00
\end{tabular}

